\title{
El problema de la determinación del contenido de cemento en morteros y hormigones fraguados
}

Prof. Dr. JOSE CALLEJA

Vicedirector del IETcc

\section{R E S U M E N}

Se trata en este trabajo de señalar el campo y las condiciones de aplicación del método NELC 5.01-a) para determinar la dosificación de cemento en morteros y hormigones fraguados, a la vista de los nuevos tipos de cemento del vigente y reciente Pliego de Prescripciones Técnicas Generales para la Recepción de Cementos (RC-75).

Se señalan los grandes errores que se pueden cometer por una inadecuada aplicación del método, y se llama la atención sobre las posibles consecuencias.

\section{ANTECEDENTES}

En varias ocasiones anteriores ha sido necesario, y en cualquier caso conveniente, tratar de la dosificación de cemento en conglomerados fraguados y endurecidos, invocando diversos aspectos del tema: unos técnicos, otros económicos, y otros legales o de responsabilidad (1) (2) (3) (4).

Con la entrada en vigor del Pliego RC-75 (5) y con la inclusión en el mismo de nuevos tipos de cementos, parece llegada una ocasión más de volver a insistir oportunamerte sobre el citado tema.

En trabajos previos se tomaron en consideración las peculiaridades propias del problema de determinar la dosificación de cemento en un hormigón, así como los fundamentos de los métodos para resolverlo y los errores y limitaciones inherentes a dichos métodos, y en particular a los practicados usualmente en nuestro país (2).

El método más utilizado suele ser el de la norma española NELC 5.01-a) (6), la cual se corresponde con la norteamericana ASTM C 85-54 y 66 (7). Este método está basado en la determinación de la sílice soluble del conglomerado, supuesta proveniente en exclusiva del conglomerante; y en el conocimiento o en la determinación del contenido de la misma en el cemento. $Y$ hay que precisar que el campo de aplicación del procedimiento se limita por lo tanto y por una parte, a morteros y hormigones que carezcan de áridos o de adiciones de materiales silícicos atacables por los ácidos; y, por otra parte - una vez salvada esta limitación-, el método es sólo aplicable al caso de conglomerados en los 
que, a pesar de no ser conocido el contenido de sílice soluble del cemento, ni disponer de muestra de éste para poder determinarlo, se tenga la evidencia de que están hechos a base de cemento portland, con objeto de poder aplicar, a fines de cálculo aproximado, un supuesto contenido de sílice soluble de dicho cemento que sea la media de los correspondientes a los cミmentos portland del país en cada instancia. Media que es aconsejable revisar periódicamente (4).

Por cemento portland se debe entender a tales efectos - y no es ocioso insistir en ello-, el constituido exclusivamentè por clínker y yeso o, en general, por clínker y retardador, sin presencia alguna de adición de cualquier tipo.

Dicho de otra forma, el método es sólo aplicable a conglomerados de cemento portland P del Pliego antiguo PCCH-64 (8); no así a los de cementos portland "comerciales" del mismo, ni a los siderúrgicos, puzolánicos, de adición, naturales o aluminosos.

Todo esto quedó cualitativa y cuantitativamente demostrado y explicado en publicaciones anteriores (2) (3), cuyas conclusiones ponían de relieve que, aparte de los errores debidos al método en sí — cuando se aplica bien-y a las hipótesis aproximativas del mismo, puede haber otros errores previsibles pero en general "imprevistos" — cuando el método se aplica mal-, los cuales pueden conducir a resultados bastante erróneos, tanto por defecto como por exceso. Por defecto, cuando el conglomerante no es cemento portland P (PCCH-64), sino cemento portland "comercial" con adiciones de tipo calizo; por exceso, cuando las adiciones son de tipo silícico — solubles en ácido clorhídrico-, tales como escorias y/o puzolanas de todo tipo. Y hasta puede no haber error "aparente" - pero sí real-, cuando la adición consista en mezclas de ciertos materiales calizos y silícicos en determinadas proporciones.

Todo esto ha llevado a proponer otros métodos de determinación de la dosificación de cemento en morteros y hormigones, los cuales se basan en los mismos principios, si bien se apoyan en la determinación de más de un componente, tanto en el cemento como en el hormigón (9) (10).

\section{ESTADO ACTUAL}

El Pliego RC-75 vigente en la actualidad (5) comtempla, además de los cementos portland P ("puros") y de los siderúrgicos, puzolánicos y demás, incluidos en el Pliego antiguo PCCH-64 (8), un nuevo tipo de cementos: el de los portland con adiciones activas, $P A$, las cuales pueden ser determinadas escorias siderúrgicas y/o determinados materiales puzolánicos naturales o artificales, incluidas las cenizas volantes.

En todo caso se trata de materiales más o menos silícico-aluminosos principalmente, y con un cierto contenido, no escaso, de sílice soluble.

Esto quiere decir que, en consonancia con lo expuesto en el punto anterior, el método NELC 5.01-a) (6) no es aplicable sino a los cementos hechos con cementos portland $\mathrm{P}$ del Pliego moderno RC-75 (5); no así a los conglomerados de cementos siderúrgicos, puzolánicos, compuestos y demás, ni tampoco a los de cementos portland con adiciones activas PA, a menos que se disponga del análisis de dichos cementos, o de una muestra de los mismos para ser analizada.

Por lo que respecta a los cementos PA, dada la naturaleza de las adiciones que contienen, y en vista de lo indicado en lo que precede, la aplicación del método NELC 5.01-a) a sus morteros y hormigones conduciría a resultados fuertemente erróneos por exceso, en el caso de no ser conocida la cuantía de sílice soluble del cemento utilizado en ellos, 
ni disponer de muestra para determinarla, y como consecuencia de ello, en el caso de utilizar indebidamente como valor medio de dicha sílice soluble el correspondiente a los cementos portland "puros" $\mathrm{P}$.

En efecto, supóngase una serie de cuatro cementos constituidos a base de un clínker con $20 \%$ de sílice soluble, y de cada una de tres puzolanas con $40 \%, 60 \%$ y $80 \%$ de sílice soluble, respectivamente, con las proporciones y con los contenidos totales de sílice soluble que se indican en el Cuadro 1.

C UADRO 1

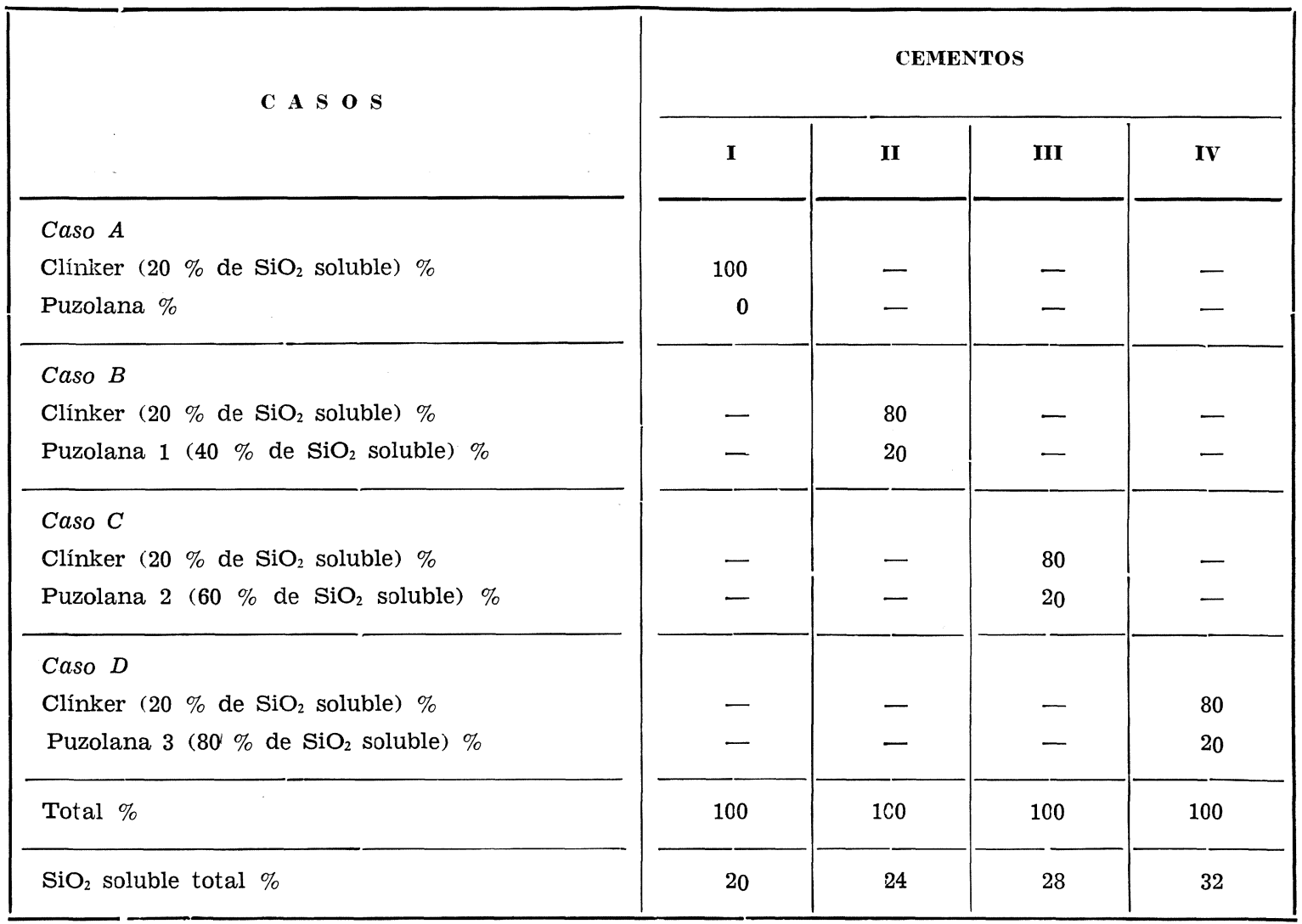

Supongan, asímismo, una serie de hormigones a base de cada uno de los cuatro cementos, con una dosificación real común de $300 \mathrm{~kg} / \mathrm{m}^{3}$, y con una densidad común de 2,25 . Admitida una aportación nula de sílice soluble por parte de los áridos, los contenidos de sílice soluble -expresados en $\mathrm{kg} / \mathrm{m}^{3}$ y en \%- de los hormigones serían los indicados en el Cuadro 2.

Por aplicación de la fórmula:

dosificación calculada $\left(\mathrm{kg} / \mathrm{m}^{3}\right)=1.000 \times 2,25 \times \frac{S_{\mathrm{h}} \%}{S_{\mathrm{c}} \%}$ en la que

$S_{\mathrm{h}} \%$ son los contenidos de sílice soluble encontrados por ánálisis en los hormigones, y $S_{\text {c }} \%$ el contenido medio de sílice soluble de los cementos portland P españoles - que en este caso se supone igual a $20 \%$-, se obtienen las dosificaciones dadas en el Cuadro 3. 


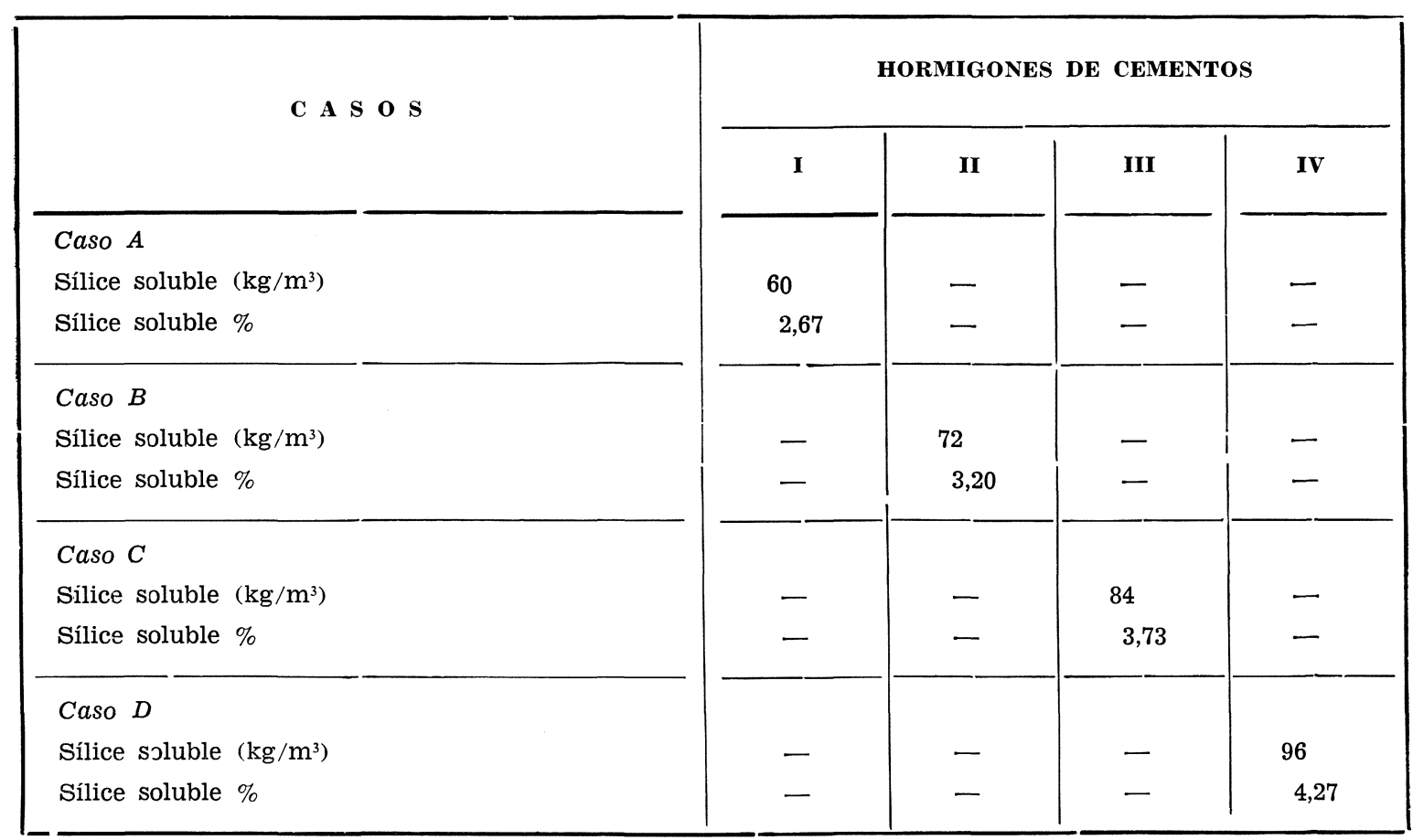

CUADRO 3

\begin{tabular}{|c|c|c|c|c|}
\hline \multirow{2}{*}{ c A s o s } & \multicolumn{4}{|c|}{ HORMIGONES DE CEMENTOS } \\
\hline & $\mathbf{I}$ & II & III & IV \\
\hline Caso $A$ & & & & \\
\hline Dosificacióa $\left(\mathrm{kg} / \mathrm{m}^{3}\right)$ & 300 & - & - & - \\
\hline$\%$ & 100 & - & - & - \\
\hline Caso $B$ & & & & \\
\hline Dosificación ( $\left.\mathrm{kg} / \mathrm{m}^{3}\right)$ & - & 360 & - & - \\
\hline$\%$ & - & 120 & - & - \\
\hline Caso $\mathrm{C}$ & & & & \\
\hline Dosificación $\left(\mathrm{kg} / \mathrm{m}^{3}\right)$ & - & - & 420 & - \\
\hline$\%$ & - & - & 140 & - \\
\hline Caso $D$ & & & & \\
\hline Dosificación $\left(\mathrm{kg} / \mathrm{m}^{3}\right)$ & - & - & - & 480 \\
\hline$\%$ & - & - & - & 160 \\
\hline
\end{tabular}

Como es de apreciar, se obtienen resultados erróneos por exceso en 20,40 y $60 \%$, para los casos B, C y D, respectivamente; es decir, son erróneos en la misma cuantía - como es natural- en que la adición incrementa en el cemento que la contiene -y por lo tanto en el hormigón-, el contenido de la sílice soluble, en relación con el valor de la misma que corresponde al cemento sin adición. 
Estos errores tan de bulto son independientes del error propio del método dimanante del hecho de que el contenido real de sílice soluble del cemento no coincida con el valor medio adoptado para el mismo. Este error puede ser por defecto, y tanto mayor cuanto mayor sea el valor del contenido medio de sílice soluble adoptado, y cuanto mayor sea la diferencia entre el contenido real de sílice soluble del cemento y dicho valor medio. También este error puede ser por exceso, y tanto mayor cuanto menor sea el valor del contenido medio de sílice soluble adoptado, y cuanto menor sea la diferencia negativa y mayor la positiva entre el contenido real de sílice soluble del cemento y dicho valor medio.

Por ejemplo, para cementos con contenidos reales de sílice soluble de 18, 19, 20 y $21 \%$, y para valores medios adoptados de 19,5 y $20,5 \%$, con el mismo hormigón de $300 \mathrm{~kg} / \mathrm{m}^{3}$ de cemento y de densidad 2,25 se tendrían las dosificaciones del Cuadro 4, correspondientes a cada uno de los casos A, B, C y D de los Cuadros 1 a 3, es decir, de cementos con 20 por ciento de cada una de las puzolanas 1,2 y 3 , que contienen 40,60 y 80 por ciento de sílice soluble, respectivamente. Las expresiones en tantos por ciento están referidas a la dosificación real y verdadera de cemento, de $300 \mathrm{~kg} / \mathrm{m}^{3}$, tomada como 100 por ciento.

C UADRO 4

\begin{tabular}{|c|c|c|c|c|c|c|c|c|c|c|}
\hline \multicolumn{11}{|c|}{ DOSIFICACIONES CALCULADAS $\mathrm{kg} / \mathrm{m}^{3}$} \\
\hline \multirow{4}{*}{\multicolumn{3}{|c|}{ C A s o s }} & \multicolumn{8}{|c|}{ Valor medio de $\mathrm{SiO}_{2}$ soluble $\%$ adoptado para los cementos } \\
\hline & & & \multicolumn{4}{|c|}{19,5} & \multicolumn{4}{|c|}{20,5} \\
\hline & & & \multicolumn{8}{|c|}{ Cementos y Hormigones } \\
\hline & & & $\mathbf{J}$ & II & III & IV & $\mathbf{I}$ & II & III & IV \\
\hline \multirow{16}{*}{ 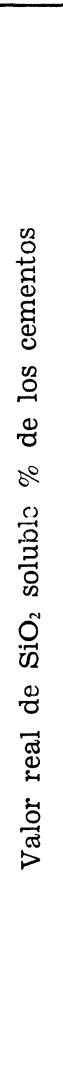 } & \multirow{4}{*}{18} & A & $\begin{array}{r}277 \\
92 \% \\
\end{array}$ & & & & $\begin{array}{r}263 \\
88 \% \\
\end{array}$ & & & \\
\hline & & B & & $\begin{array}{ll}338 \\
113 \% \\
\end{array}$ & & & & $\begin{array}{l}322 \\
107 \%\end{array}$ & & \\
\hline & & $\mathbf{C}$ & & & $\begin{array}{ll}406 \\
135 \% \\
\end{array}$ & & & & $\begin{array}{l}386 \\
129 \%\end{array}$ & \\
\hline & & $\mathrm{D}$ & & & & $\begin{array}{ll}467 & \\
156 \% \\
\end{array}$ & & & & $\begin{array}{l}445 \% \\
148 \%\end{array}$ \\
\hline & \multirow{4}{*}{19} & A & $\begin{array}{r}292 \\
97 \% \\
\end{array}$ & & & & $\begin{aligned} & 278 \\
& 93 \% \\
&\end{aligned}$ & & & \\
\hline & & B & & $\begin{array}{l}356 \\
119 \% \\
\end{array}$ & & & & $\begin{array}{l}339 \\
113 \%\end{array}$ & & \\
\hline & & $\mathbf{C}$ & & & $\begin{array}{ll}419 & \\
140 \% \\
\end{array}$ & & & & $\begin{array}{l}398 \\
133 \%\end{array}$ & \\
\hline & & $\mathrm{D}$ & & & & $\begin{array}{ll}480 & \\
160 \% \\
\end{array}$ & & & & $\begin{array}{ll}457 \\
152 \% \\
\end{array}$ \\
\hline & \multirow{4}{*}{20} & A & $\begin{array}{l}308 \% \\
103 \% \\
\end{array}$ & & & & $\begin{array}{r}293 \\
98 \% \\
\end{array}$ & & & \\
\hline & & B & & $\begin{array}{l}369 \\
123 \%\end{array}$ & & & & $\begin{array}{ll}351 & \\
117 \% \\
\end{array}$ & & \\
\hline & & $\mathbf{C}$ & & & $\begin{array}{l}430 \\
143 \%\end{array}$ & & & & $\begin{array}{l}.109 \\
136 \%\end{array}$ & \\
\hline & & $\mathrm{D}$ & & & & $\begin{array}{l}493 \\
164 \% \\
\end{array}$ & & & & $\begin{array}{ll}469 & \\
156 \% \\
\end{array}$ \\
\hline & \multirow{4}{*}{21.} & A & $\begin{array}{l}323 \% \\
108 \% \\
\end{array}$ & & & & $\begin{array}{l}307 \% \\
102 \% \\
\end{array}$ & & & \\
\hline & & B & & $\begin{array}{l}382 \\
127 \%\end{array}$ & & & & $\begin{array}{l}363 \% \\
121 \%\end{array}$ & & \\
\hline & & $\mathrm{C}$ & & & $\begin{array}{l}443 \\
148 \% \\
\end{array}$ & & & & $\begin{array}{ll}421 & \\
140 \% \\
\end{array}$ & \\
\hline & & $\mathrm{D}$ & & & & $\begin{array}{l}504 \% \\
168 \%\end{array}$ & & & & $\begin{array}{l}480 \\
160 \%\end{array}$ \\
\hline
\end{tabular}


Como se deduce del Cuadro 4, los errores por exceso pueden oscilar entre el 2 y el 68 por ciento - casos 21 A I 20,5 y 21 D IV 19,5-, mientras que los errores por defecto pueden oscilar entre el 2 y el 12 por ciento - casos 20 A I 20,5 y 18 A I 20,5-.

\section{CONCLUSIONES}

A la vista de lo que precede, es lícito extraer las siguientes conclusiones:

1. ${ }^{a} \quad$ El método NELC 5.01-a) (6) para la determinación del contenido de cemento portland en un conglomerado (mortero u hormigón) fraguado, es aplicable a todos los cementos del Pliego RC-75, a base de clínker portland, siempre que se conozca el contenido de sílice soluble del cemento, o se disponga de una muestra representativa del mismo para determinar dicho contenido de sílice soluble.

2. ${ }^{a} \quad$ Cuando no se conoce el contenido de sílice soluble del cemento ni se dispone de muestras del mismo para determinar dicho contenido, el método NELC 5.01-a) sólo es aplicable a los conglomerados hechos con cementos portland $\mathrm{P}$ del Pliego RC-75, tomado - por el momento- como valor medio del contenido de sílice soluble de dichos cementos el de 19,5 por ciento (4).

3. ${ }^{a}$ La aplicación injustificada e incorrecta del método NELC 5.01-a) en la forma indicada, a conglomerados hechos con cementos distintos de los portland P del Pliego RC75, y en particular a los hechos con cementos portland con adiciones activas PA de dicho Pliego, conduciría a resultados altamente erróneos por exceso e inadmisibles, con el consiguiente riesgo grave de hacer hipótesis o de establecer conclusiones falsas sobre bases absolutamente irreales.

4. ${ }^{a}$ Lo precisado en las conclusiones anteriores quiere decir que, si por cualquier circunstancia no se utilizaran cementos portland $\mathrm{P}$, cualquier cuestión relativa a la dosificación de cemento en hormigones exigiría imprescindiblemente conocer el análisis del cemento, o disponer de una muestra del mismo para efectuarlo.

$5 .^{a} \quad \mathrm{Si}$ por equivocación -o por el motivo que fuere- se envasase y expidiese cemento $\mathrm{PA}$ en bolsas o contenedores con el marchamo correspondiente a cemento $\mathrm{P}$, y como tal se utilizase, la aplicación -aparentemente correcta en este caso, pero totalmente inadmisible- del método NELC 5.01-a) (6) daría lugar a los fuertes errores por exceso señalados en el presente trabajo.

\section{R E F E R E N C I A S}

(1) Calleja, J.: "Determinación de la dosis de cemento en hormigones y morteros endurecidos". I Seminario de Construcción de Carreteras (IETCC), Madrid 1961.

(2) Calleja, J.: "Observaciones acerca de la dosificación de cementos en morteros y hormigones iraguados”. Materiales de Construcción (IETCC) núm. 121 (1966).

(3) Calleja, J.: "Consideraciones sobre los contenidos nominal y real de materiales hidráulicamente activos en los conglomerados”. Materiales de Construcción (IETCC) núm. 122 (1966).

(4) Calleja, J. y del Olmo, C.: "Determinación del contenido de cemento en morteros y hormigones îraguados: datos para la puesta al día del método". Materiales de Construcción (IETCC) núm. 140 (1970).

(5) Pliego RC-75: "Pliego de Prescripciones Técnicas Generales para la Recepción de Cementos". MOP Madrid 1975. 
(6) NELC 5.01-a): Normas de Ensayo del Laboratorio Central de Ensayo de Materiales de Construcción. Publicación 42: "Determinación de la dosificación de cemento de los morteros y hormigones fraguados fabricados con cemento". Madrid 1947.

(7) ASTM Standards: Métodos 85-54 y 85-66: "Cement content of hardened portland cement concrete". 1961 .

(8) PCCH-64: Pliego General de Condiciones para la Recepción de Conglomerantes Hidráulicos en Obras de Carácter Oficial. Normas y Manuales (IETCC), Madrid 1964.

(9) RuIz DE GaUnA, A.: "La determinación de la dosificación de cemento de los morteros y hormizones fraguados, en los casos fuera del ámbito de aplicación de los métodos usuales para hallarla". Materiales de Construcción (IETCC), núm. 121 (1966).

(10) RuIz DE Gauna, A.: Tesis doctoral dirigida por J. Calleja: "Contribución al estudio de los morteros y hormigones fraguados: nuevo procedimiento de dosificación con aplicación de computadores digitales". Madrid 1970. 